This is the peer reviewed version of the following article: Amaral, S., Tawara, M., FernandezVillamarin, M., Borrajo, E., Martínez-Costas, J., \& Vidal, A. et al. (2018). Tuning the Size of Nanoassembles: A Hierarchical Transfer of Information from Dendrimers to Polyion Complexes. Angew. Chem. Int. Ed., 57(19), 5273-5277, which has been published in final form at https:// doi.org/10.1002/anie.201712244. This article may be used for non-commercial purposes in accordance with Wiley Terms and Conditions for Use of Self-Archived Versions 


\title{
Tuning the Size of Nanoassembles: A Hierarchical Transfer of Information from Dendrimers to Polyion Complexes
}

\author{
Sandra P. Amaral, Maun H. Tawara, Marcos Fernandez-Villamarin, Erea Borrajo, José Martínez- \\ Costas, Anxo Vidal, Ricardo Riguera, and Eduardo Fernandez-Megia*
}

\begin{abstract}
Herein we describe that the generation of dendrimers represents a powerful tool in the control of the size and biodistribution of polyion complexes (PIC). Using a combinatorial screening of six dendrimers (18-243 terminal groups) and five oppositely charged PEGylated copolymers, a dendrimer to PIC hierarchical transfer of structural information was revealed with PIC diameters that increased from 80 to $500 \mathrm{~nm}$ on decreasing the dendrimer generation. This rise in size, also accompanied by a micelle to vesicle transition, is interpreted according to a cone- to rod-shaped progression in the architecture of the unit PIC (UPIC). This precise size tuning entitled dendritic PIC as nanorulers for controlled biodistribution. Overall, a domino-like control of the size and biological properties of PIC -not attainable with linear polymers- is feasible through dendrimer generation.
\end{abstract}

Since modern chemistry and the atomic theory were introduced, a handful of profound breakthroughs have dramatically contributed to our understanding of the complex organization of matter. ${ }^{1} \mathrm{~A}$ hierarchical transfer of structural information has been revealed from atoms with discrete atomic numbers and electronic configurations to complex molecules, macromolecules and bio-assemblies at the origin of life. ${ }^{1,2}$ Using the same rationale in the search for innovative functions, scientists attempted to mimic nature by engineering supramolecular structures. ${ }^{3,4}$ In the quest for a systematic framework for unifying nanoscience as a critical piece between atoms and macroscopic matter, Tomalia has recently proposed dendrimers as soft superatoms: quantized building blocks

["] S. P. Amaral, M. H. Tawara, Dr. M. Fernandez-Villamarin, Prof. Dr. R. Riguera, Prof. Dr. E. Fernandez-Megia Centro Singular de Investigación en Química Biolóxica e Materiais Moleculares (CIQUS) and Departamento de Química Orgánica Universidade de Santiago de Compostela

Jenaro de la Fuente s/n, 15782 Santiago de Compostela, Spain. E-mail: ef.megia@usc.es

Dr. E. Borrajo, Prof. Dr. A. Vidal

Departamento de Fisioloxía and Centro Singular de Investigación en Medicina Molecular e Enfermidades Crónicas (CIMUS) Universidade de Santiago de Compostela; Instituto de Investigaciones Sanitarias de Santiago de Compostela (IDIS) 15782 Santiago de Compostela, Spain.

Prof. Dr. J. Martínez-Costas

Centro Singular de Investigación en Química Biolóxica e Materiais Moleculares (CIQUS) and Departamento de Bioquímica e Bioloxía Molecular

Universidade de Santiago de Compostela

15782 Santiago de Compostela, Spain.

Supporting information for this article is given via a link at the end of the document. mirroring the role of atoms at the picoscale.1,5 Dendrimers are well-defined, monodisperse, and globular macromolecules that, in contrast to traditional polymers, are synthesized with precise control over six critical nanoscale design parameters: size (1-20 $\mathrm{nm}$ ), shape, surface chemistry, flexibility/rigidity, architecture, and elemental composition. ${ }^{6}$ In this way, the dendritic structure is sought to steer supramolecular associations towards more complex, ordered matter. ${ }^{1,7,8,9,10,11}$ Herein, we describe that the information stored within the size and generation $(G)$ of dendrimers can be hierarchically transferred to polyion complexes $(\mathrm{PIC})^{12,13,14}$ and determine in a domino-like fashion -not attainable with linear polymers- their size and in vivo biodistribution.

PIC micelles and vesicles (PICsomes) are nanoassembles prepared from oppositely charged polymers and PEGylated block copolymers. ${ }^{15,16,17}$ Although attracting much attention as drug delivery systems (DDS) because of their electrical neutrality, stealth character and narrow size distribution, an extremely high salt-sensitivity still represents a challenge for PIC. To overcome this shortcoming, we have recently described PIC micelles from PEG-dendritic block copolymers. ${ }^{18,19}$ The intrinsic rigidity and globular nature of a dendritic (GATG=gallic acid-triethylene glycol) block ${ }^{20,21,22}$ has resulted in micelles with unprecedented stability in serum and to ionic strengths higher than $3 \mathrm{M}$, the highest achieved for PIC.

The pharmacokinetics and biodistribution of DDS depend on size. ${ }^{23}$ Although size control in PIC has been somewhat achieved by adjusting the composition and concentration of charged linear polymers, ${ }^{24,25}$ a more straightforward approach based on molecular weight (MW) has been hampered by a limited response of PIC. ${ }^{26}$ As an alternative, we envisaged the use of charged dendrimers, where information stored within $G$ might be hierarchically transferred to PIC with oppositely charged PEGylated linear copolymers, and tune their size and biological properties. We stand our hypothesis on the large differences in local dynamics between linear polymers (dependent on segment, not MW) and dendrimers (that depend on $\mathrm{G}$ and hence, MW). ${ }^{27}$ Since such a size tuning has not been unveiled so far in dendritic PIC micelles ${ }^{28,29,30}$ and vesicles ${ }^{31}$, we decided to thoroughly explore the scope of a dendrimer to PIC hierarchical transfer of information using a combinatorial approach comprising a large PIC library from six GATG amino-dendrimers (18-243 terminal amines) and five PEG-PGA (poly-L-glutamic acid) linear copolymers with various PEG (5 and $20 \mathrm{kDa}$; corresponding to a degree of polymerization DP 113 and 455 , respectively) and PGA (DP 25, 50, 100, 200) blocks lengths. 


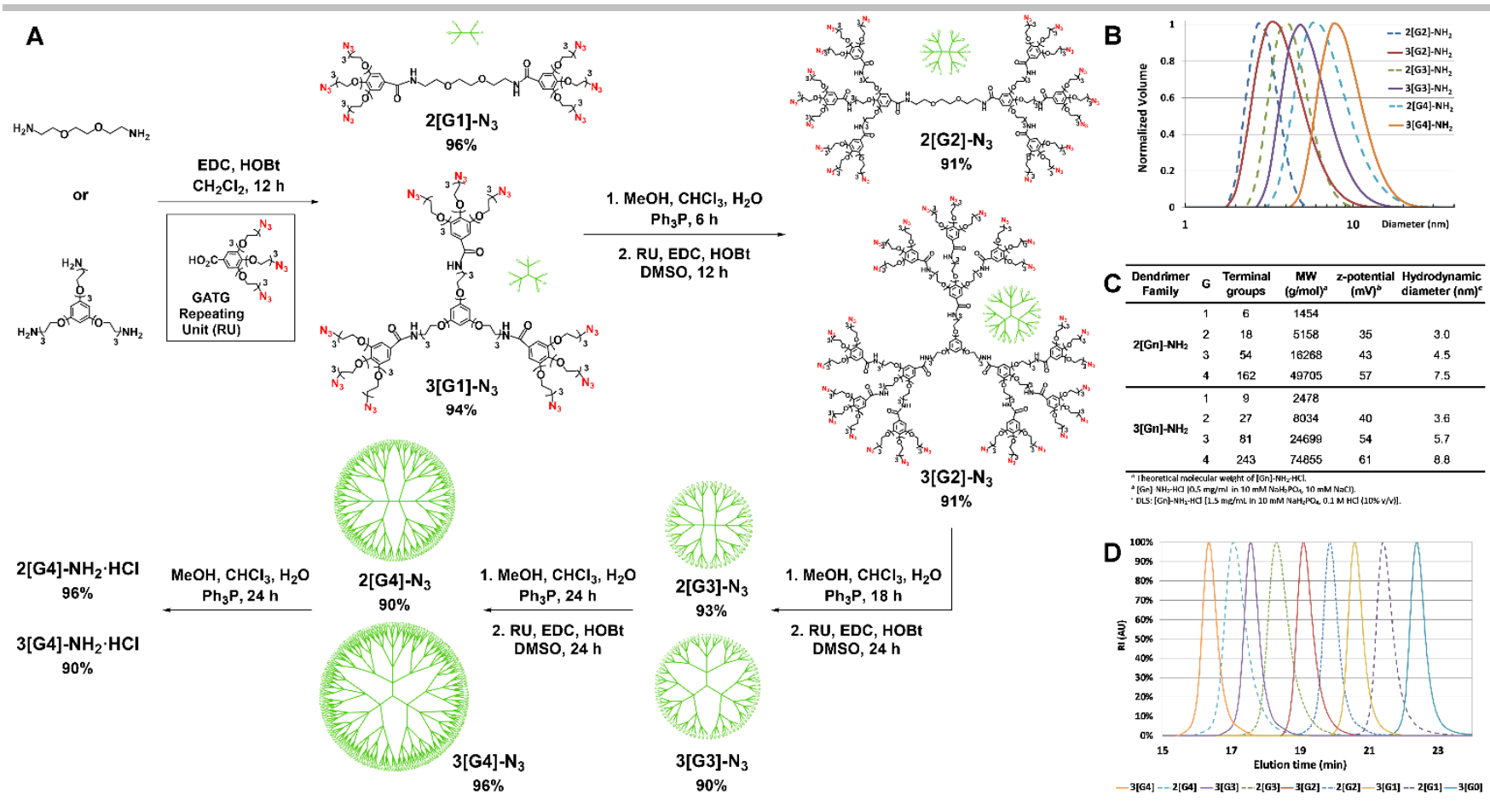

Figure 1. Synthesis of $2[\mathrm{Gn}]$ and $3[\mathrm{Gn}]$ dendrimers $(A)$. DLS size distribution of $[\mathrm{Gn}]-\mathrm{NH}_{2} \cdot \mathrm{HCl}(B)$. Structural data of dendrimers $(C)$. GPC elugrams of $[\mathrm{Gn}]-\mathrm{N}_{3}$ (THF) (D).

With this aim, two new families of symmetrical GATG dendrimers, namely $2[\mathrm{Gn}]$ and $3[\mathrm{Gn}]$, were designed from di- and trifunctionalized cores (Figure 1). Following a divergent growth sequence involving an amide coupling with the GATG repeating unit (EDC, HOBt, 93-96\%) and azide reduction $\left(\mathrm{Ph}_{3} \mathrm{P}, 90-100 \%\right)$, four dendrimer generations were synthesized in excellent yields with up to 243 terminal azide/amino groups: 2[G1] (6 terminal groups), 3[G1] (9), 2[G2] (18), 3[G2] (27), 2[G3] (54), 3[G3] (81), 2[G4] (162), 3[G4] (243). The high solubility of these dendrimers facilitates their preparation at high $\mathrm{G}$ and purification in large scale (MPLC in just $10 \mathrm{~min}$, ultrafiltration is also possible for G3 and G4) the bottlenecks in the synthesis of dendrimers. Their chemical characterization is described in the SI with convincing evidence by ${ }^{1} \mathrm{H}$ and ${ }^{13} \mathrm{C}$ NMR, IR and MALDI-TOF MS. In addition, their purity and monodispersity were verified by gel permeation chromatography (GPC) and dynamic light scattering (DLS) that showed the expected increased in size with $\mathrm{G}$ (Figure 1).

The formation of PIC was straightforward by mixing in stoichiometric charge ratios the G2-G4 amino-dendrimers (18243 terminal amines) and PEG-PGA (PEG ${ }_{5 k}-P A_{25}, P E G_{5 k}-$ $\left.P A_{50}, P E G_{5 k}-P G A_{100}, P E G_{20 k}-P G A_{100}, P E G_{20 k}-P G A_{200}\right)$ in $10 \mathrm{mM}$ phosphate buffer (PB), supplemented with $150 \mathrm{mM} \mathrm{NaCl}$ (Figure 2). DLS measurements performed $2 \mathrm{~h}$ after mixing confirmed the presence of PIC with low polydispersity and hydrodynamic diameters in the $80-500 \mathrm{~nm}$ range. PIC revealed high stability over time and temperature (dialysis in $10 \mathrm{mM} \mathrm{PB} \mathrm{pH} \mathrm{7.4,} 150 \mathrm{mM} \mathrm{NaCl}$ for $24 \mathrm{~h}$ at $25^{\circ} \mathrm{C}$, followed by $24 \mathrm{~h}$ at $37^{\circ} \mathrm{C}$ ), even for dendrimers as small as 2[G2] with only 18 terminal amines (Figures 2 and S3S6, Table S1); exceeding the stability of PIC from linear polymers of much higher DP as previously reported. ${ }^{18,19}$ Larger dendrimers afforded smaller PIC, an effect associated to their higher rigidity and multivalency, in accordance with our hypothesis of a hierarchical transfer of information. No PIC was obtained from $P E G_{5 k}-P G A_{25}$. Nevertheless, increasing the length of the PGA block successfully led to discrete PIC with G3-G4 for PEG $\mathrm{k}^{-}$ $P G A_{50}$, and with all dendrimers for $P E G_{5 k}-P G A_{100}$; pointing to more stable PIC from the largest dendrimers and PGA blocks. The formation efficiency of these PIC was determined as ca. 94\% by quantifying the amount of free dendrimer in solution after ultracentrifugation of the 2[G3]/PEG ${ }_{5 k}-P A_{100} P I C(S I)$. Despite the high z-potential of the amino-dendrimers (Figure 1), values for PIC were close to zero in agreement with the PEG corona and the charge stoichiometry of the constituents (SI).

Next, a detail structural analysis was performed with four PIC samples selected from the $P E_{5 k}-P G A_{100}$ series, the one affording the largest size variation (PIC with 2[G4], 3[G3], 2[G3], and 3[G2] with sizes ca. 100, 175, 200 and 450 nm; Figure 3). Gratifyingly, AFM studies revealed mean diameters in agreement with DLS measurements: 2[G4] $(87 \pm 7 \mathrm{~nm}), 2[\mathrm{G} 3](222 \pm 17 \mathrm{~nm})$, $3[\mathrm{G} 2](435 \pm 34 \mathrm{~nm})$. Analysis by cryo-TEM showed the 2[G4] PIC as micelle and the three larger assembles as PICsomes with uniform lamella of thickness $22.19 \pm 1.41 \mathrm{~nm}$ for $3[\mathrm{G} 3$ ], $13.50 \pm 0.59$ $\mathrm{nm}$ for 2[G3], and 22.12 $\pm 2.38 \mathrm{~nm}$ for 3[G2]. With all PIC in the $P E G_{5 k}-P G A_{100}$ series having an identical PEG weight fraction (ca. $13 \%$ ), we interpret their G-dependent size and structure based on the architecture of the monomeric unit PIC (UPIC, the minimum PIC assemble generated in the early stages of the PIC growth) ${ }^{32}$ We hypothesize that the size and rigidity of the dendrimer component determine the architecture of the UPIC, which in turn governs the size and structure of the final PIC assemble. Thus, small and flexible dendrimers might easily adopt rod-like UPIC with $P E G_{5 k}-P G A_{100}$ that accommodate into $2 D$ lamellae, therefore producing PICsomes. On the contrary, larger and more rigid 
A
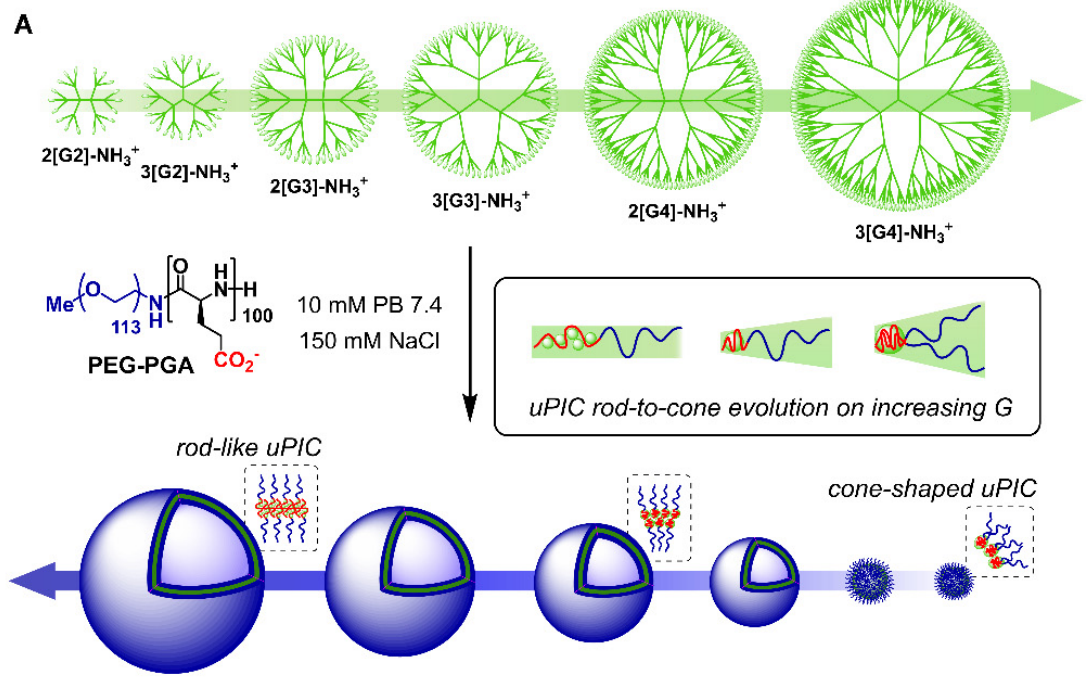
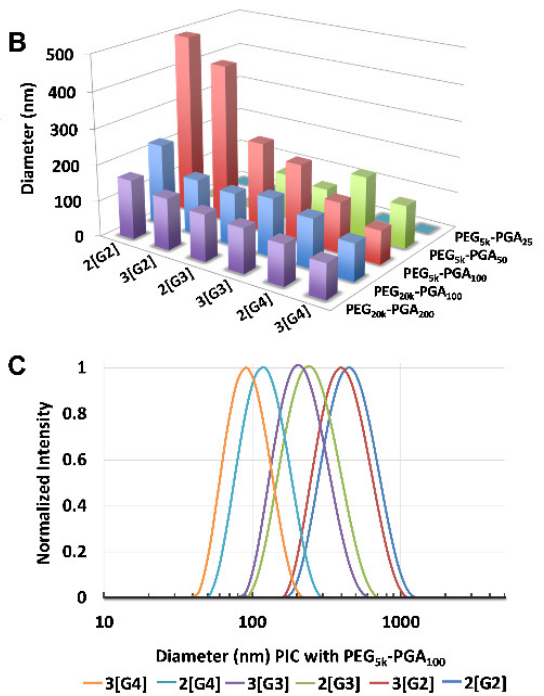

Figure 2. Schematic representation of the dendrimer to PIC hierarchical transfer of structural information (A). DLS mean diameters (B) and size distribution (C) of $\mathrm{PIC}$ after $24 \mathrm{~h}$ at $37^{\circ} \mathrm{C}$ in $10 \mathrm{mM} \mathrm{PB}, \mathrm{pH} 7.4,150 \mathrm{mM} \mathrm{NaCl}$

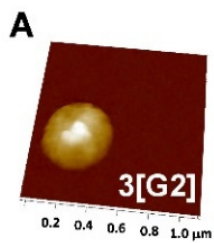

B
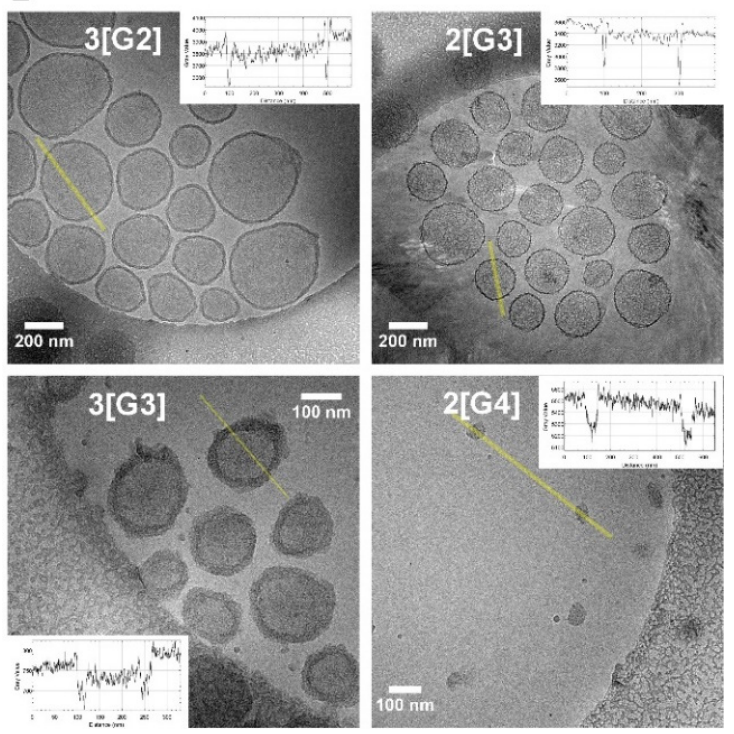

Figure 3. AFM (A) and cryo-TEM (B) images of PICsomes (3[G2], 2[G3], 3[G3]) and PIC micelles (2[G4]) from PEG $5 k-P G A_{100}$.

dendrimers afford cone-shaped UPIC leading to micelles (Figure 2). The larger PIC sizes and micelle to vesicle transition observed on decreasing the dendrimer $G$ are also explained by $a$ progressive cone to rod evolution in the architecture of the UPIC. Thus, within the PICsome window, the more rod-like character of the UPIC (associated to smaller dendrimers), the better its fitting into lamellae with smaller curvature leading to larger vesicles. ${ }^{12}$
The same applies to PIC from large dendrimers in the micelle window: the more cone-shaped UPIC on increasing the dendrimer $G$ affords smaller micelles. As a result, the architecture of the UPIC emerges as a valuable tool in analyzing the size and predicting the structure (micelle to vesicle transition) of PIC. The same model explains the smaller PIC observed from $\mathrm{PEG}_{20 \mathrm{k}}$ copolymers. The larger PEG block in $P E G_{20 k}-P G A_{100}$ compared to $P E G_{5 k}-P G A_{100}$ should render more cone-shaped dendritic UPIC, favoring the formation of smaller assembles. Finally, not unexpectedly, the MW of the PGA block plays a less drastic role on the size of this PIC library.

The cell internalization and toxicity profile of these PIC were investigated in human adenocarcinoma alveolar basal epithelial (A549) cells with the $100 \mathrm{~nm}$ PIC (2[G4]/PEG Fk $\left.-P A_{100}\right)$. A MTT assay revealed limited effect of PIC on cell proliferation (Figure $4 \mathrm{~A})$. Even at the highest concentrations analysed, the toxicity associated to the cationic amino-dendrimer was completely screened within the PIC, proving their integrity in culture medium supplemented with fetal bovine serum (FBS). Cellular uptake was studied by laser scanning confocal microscopy (LSCM) with PIC fluorescently labelled at the amino groups of the dendrimer with the green dye fluorescein isothiocyanate (FITC) (SI). Figure 4B shows images of A549 cells treated for as little as 10 min with FITC-PIC (green) that after cell uptake completely colocalize with LysoTracker Red (red), a well-known endosome/lysosome marker (nuclei stained in blue with Hoechst). Interestingly, control experiments performed at the same time with 2[G4]-FITC dendrimer showed entirely different internalization kinetics, with almost no dendrimer found inside the cell while bound to the cell membrane by electrostatic interactions. Such a different localization pattern confirms internalization of the intact PIC without disassembling in the extracellular media supplemented with FBS (validation of this result was also obtained via dual labelling experiment with FITC at the dendrimer and rhodamine $B$ at PEG-PGA, Figure S12). The final fate of PIC in endosome/lysosome, organelles with a characteristic acidic/enzymatic environment, encourages their use as DDS for intracellular drug release following internalization by endocytosis. 

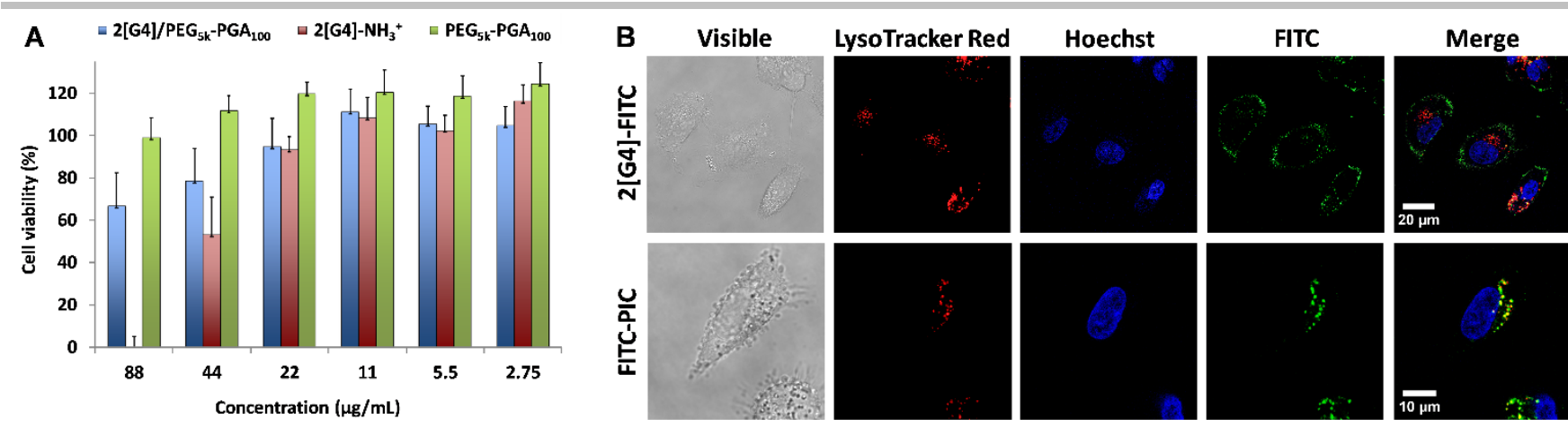

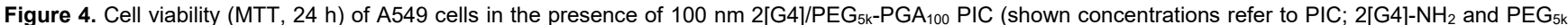
$\mathrm{PGA}_{100}$ were used as controls at the same concentrations as in PIC) (A). LSCM images of A549 cells incubated for 10 min with FITC-PIC and control dendrimer 2[G4]-FITC (B)

Considering the relevance of size on the pharmacokinetics and biodistribution of DDS, we envisioned these PIC assembles as privileged nanorulers for a controlled biodistribution in vivo by near infra-red (NIR) fluorescence, using Cyanine7 (Cy7) as dye. To this end, the $100 \mathrm{~nm}$ (2[G4]/PEG $\left.{ }_{5 k}-P G_{100}\right), 200 \mathrm{~nm}$ (2[G3]/PEG $\left.{ }_{5 k}-P \mathrm{AA}_{100}\right)$ and $450 \mathrm{~nm}\left(3[\mathrm{G} 2] / \mathrm{PEG}_{5 k}-\mathrm{PGA}_{100}\right) \mathrm{PIC}$ were crosslinked and labelled with Cy7-NHS ester at the amino groups of the dendrimers (Figures S7-S9 and S13). After intravenous injection in the tail vein of nude mice, NIR images of excised organs were obtained upon sacrifice at different time points $(1,8,24$ and $72 \mathrm{~h})$. As shown in Figures 5 and S14-S17, high fluorescence intensity was found in highly vascularized organs such as liver and spleen, which also count with resident macrophages that can contribute to uptake. Apart from this, the three PIC show a differential pattern of biodistribution depending on size. The smaller $100 \mathrm{~nm}$ micelle displayed faster kinetics with a broad organ distribution as soon as $1 \mathrm{~h}$ after injection, including lung, one of the predominant sites of metastatic disease. Interestingly, they could also be detected in the lymph nodes, a finding that opens the venue for possible drug delivery directed to lymphatic dissemination of advanced cancer. Organ fluorescence at the longest time point assessed $(72 \mathrm{~h}$ ) corresponded also to the $100 \mathrm{~nm}$ PIC, a finding consistent with previous observations where nanosystems in the range of $100 \mathrm{~nm}$ prove long-lasting in circulation. ${ }^{23,24,25}$ In contrast, the largest $450 \mathrm{~nm}$ PIC showed preferential accumulation in liver, kidneys and spleen, while poorly localized in lymph nodes or absent of organs such as heart, where small PIC were readily detected. The persistent (from 1 to $72 \mathrm{~h}$ ) signal found in kidneys for the $450 \mathrm{~nm}$ PIC probably reflects an impaired excretion due to their size. These results show that a G-dependent modulation of size in dendritic PIC allows differential organ distribution and kinetics. This distinct accumulation is envisaged with great potential in delivery applications. By tailoring size, not only treatments could be preferentially directed to certain tissues, but also minimize their delivery to particular organs to prevent toxicity associated to drug treatments.

In conclusion, a dendrimer to PIC hierarchical transfer of structural information is reported, where the dendrimer $G$ controls the size and biodistribution of PIC. By decreasing the dendrimer G, larger PIC sizes (from 80 to $500 \mathrm{~nm}$ ) accompanied by a micelle to vesicle transition were observed, which is interpreted according to a cone- to rod-shaped progression in the architecture of the UPIC (the minimum PIC assembles involved in PIC growth). This precise size tuning entitled dendritic PIC as nanorulers for controlled biodistribution: a $100 \mathrm{~nm}$ PIC showed faster kinetics, broader organ distribution and longer circulation times than larger assembles. Overall, a domino-like control of the size and biological properties of PIC -not attainable with linear polymersis shown through dendrimer generation. This result along the previously reported superior stability of dendritic PIC encourage further exploration of their applications in biomedicine.
A

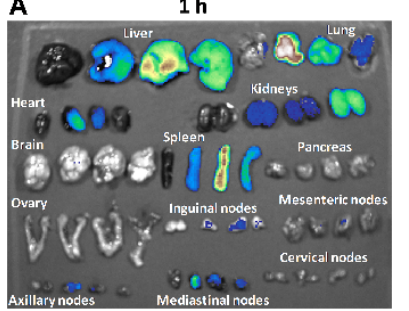

B

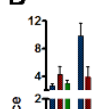

$1 \mathrm{~h}$

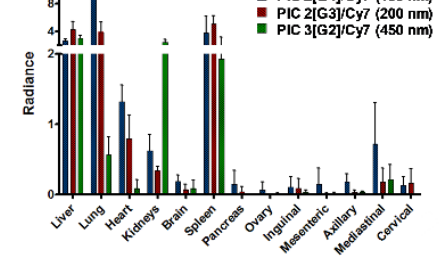

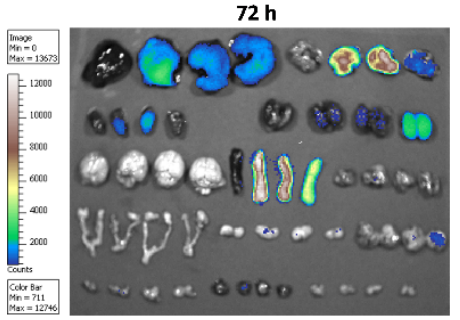

$72 \mathrm{~h}$
Figure 5. IVIS fluorescence images of selected organs $1 \mathrm{~h}$ (left) and $72 \mathrm{~h}$ (right) after injection. From left to right organs correspond to non-injected animals (control), and mice injected with Cy7-labelled PIC of 100, 200 and $450 \mathrm{~nm}$ (A). Quantification of fluorescence (radiance, photons $/ \mathrm{s} / \mathrm{cm}^{2} / \mathrm{sr} \times 10^{7}$ ) in excised organs $1 \mathrm{~h}$ (left) and $72 \mathrm{~h}$ (right) after injection of PIC (data represents mean \pm SEM, n=3) (B).

\section{Acknowledgements}

This work was financially supported by the Spanish Ministry of Economy, Industry and Competitiveness (MINECO) (CTQ201569021-R, CTQ2014-61470-EXP, BFU2013-43513-R), the Xunta de Galicia (GRC2014/040 and Centro Singular de Investigación de Galicia Accreditation 2016-2019, ED431G/09) and the European Union (European Regional Development Fund-ERDF). 
Keywords: dendrimer $\bullet$ polyion complex $\bullet$ PIC $\bullet$ micelle $\bullet$ vesicle

- PICsome $\cdot$ hierarchical transfer

1 D. A. Tomalia, S. N. Khanna, Chem. Rev. 2016, 116, 2705.

2 A. Lima-de-Faria, BioSystems 1997, 43, 115.

3 J.-F. Lutz, J.-M. Lehn, E. W. Meijer, K. Matyjaszewski, Nat. Rev. Mater. 2016 $1,16024$.

4 S. Erbas-Cakmak, D. A. Leigh, C. T. McTernan, A. L. Nussbaumer, Chem. Rev. 2015, 115, 10081

5 J. Kemsley, Chem. Eng. News 2013, 91, 24

6 D. A. Tomalia, J. Nanopart. Res. 2009, 11, 1251.

7 D. A. Tomalia, Soft Matter 2010, 6, 456

8 M. A. Kostiainen, O. Kasyutich, J. J. L. M. Cornelissen, R. J. M. Nolte, Nature Chem. 2010, 2, 394.

9 B. M. Rosen, C. J. Wilson, D. A. Wilson, M. Peterca, M. R. Imam, V. Percec Chem. Rev. 2009, 109, 6275

10 B. N. S. Thota, L. H. Urner, R. Haag, Chem. Rev. 2016, 116, 2079

11 S. E. Sherman, Q. Xiao, V. Percec, Chem. Rev. 2017, 117, 6538

12 A. Koide, A. Kishimura, K. Osada, W. D. Jang, Y. Yamasaki, K. Kataoka, J. Am. Chem. Soc. 2006, 128, 5988.

13 A. Harada, K. Kataoka, Macromolecules 1995, 28, 5294

14 A. V. Kabanov, S. V. Vinogradov, Y. G. Suzdaltseva, V. Y. Alakhov, Bioconjugate Chem. 1995, 6, 639.

15 H. Yoon, E. J. Dell, J. L. Freyer, L. M. Campos, W.-D. Jang, Polymer 2014 $55,453$.

16 A. Kishimura, Polym. J. 2013, 45, 892.

17 Y. Lee, K. Kataoka, Soft Matter 2009, 5, 3810.

18 M. Fernandez-Villamarin, A. Sousa-Herves, S. Porto, N. Guldris, J. MartinezCostas, R. Riguera, E. Fernandez-Megia, Polym. Chem. 2017, 8, 2528.

19 A. Sousa-Herves, E. Fernandez-Megia, R. Riguera, Chem. Commun. 2008 3136.

20 M. Fernandez-Villamarin, A. Sousa-Herves, J. Correa, E. M. Munoz, P. Taboada, R. Riguera, E. Fernandez-Megia, ChemNanoMat 2016, 2, 437

21 A. Sousa-Herves, C. Sánchez Espinel, A. Fahmi, Á. González-Fernández, E. Fernandez-Megia, Nanoscale 2015, 7, 3933.

22 A. Sousa-Herves, R. Novoa-Carballal, R. Riguera, E. Fernandez-Megia, AAPS J. 2014, 16, 948.

23 E. Blanco, H. Shen, M. Ferrari, Nat. Biotechnol. 2015, 33, 941.

24 H. Cabral et al., Nat. Nanotech. 2011, 6, 815.

25 Y. Anraku, A. Kishimura, A. Kobayashi, M. Oba, K. Kataoka, Chem. Commun 2011, 47, 6054.

26 H. M. van der Kooij, E. Spruijt, I. K. Voets, R. Fokkink, M. A. Cohen Stuart J. van der Gucht, Langmuir 2012, 28, 14180 and references therein.
27 L. F. Pinto, J. Correa, M. Martin-Pastor, R. Riguera, E. Fernandez-Megia, J. Am. Chem. Soc. 2013, 135, 1972.

28 J. Wang, I. K. Voets, R. Fokkink, J. van der Gucht, A. H. Velders, Soft Matter 2014, 10, 7337

29 F. Reinhold, U. Kolb, I. Lieberwirth, F. Gröhn, Langmuir 2009, 25, 1345.

30 Y. Li et al., Chem. Mater. 2007, 19, 5557.

31 K. Naoyama, T. Mori, Y. Katayama, A. Kishimura, Macromol. Rapid Commun. 2016, 37, 1087

32 Y. Anraku, A. Kishimura, Y. Yamasaki, K. Kataoka, J. Am. Chem. Soc. 2013 135, 1423.

\section{Table of Contents}
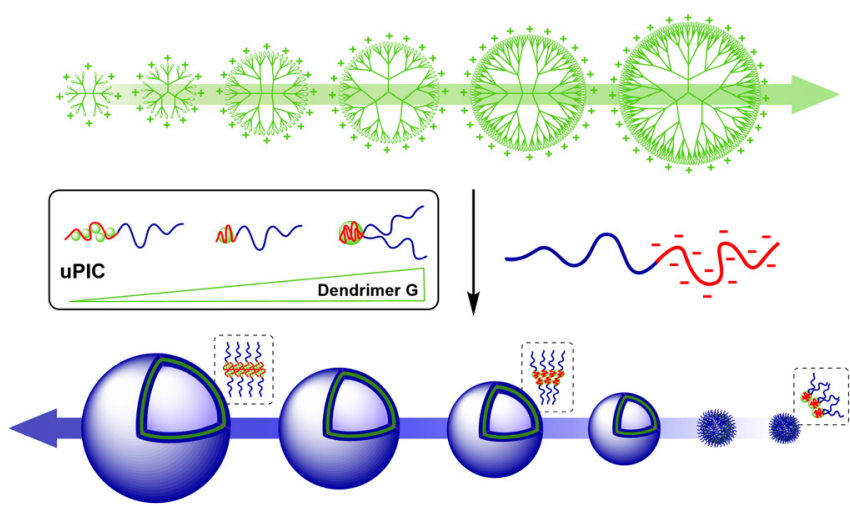

The size and biodistribution of polyion complexes (PIC) can be hierarchically tuned with the generation of a dendrimer component in a way not attainable with linear polymers. 